\title{
ATENDIMENTO AOS PROFISSIONAÍS VÍTIMAS DE ACIDENTE COM MATERIAL BIOLÓGICO EM UM HOSPITAL DE DOENÇAS INFECTOCONTAGIOSAS
}

\author{
ATTENDING PROFESSIONALS VICTIMS OF ACCIDENT WITH BIOLOGICAL MATERIAL IN A TROPICAL \\ DISEASES HOSPITAL
}

ATENDIMIENTO A LOS PROFESIONALES VÍTIMAS DE ACCIDENTE CON MATERIAL BIOLÓGICO EN UM HOSPITAL DE ENFERNEDADES TROPICAIS

\author{
Lillian Kelly de Oliveira Lopes ${ }^{1}$ \\ Anaclara Ferreira Veiga Tipple ${ }^{2}$ \\ Sirlene Neves Damando ${ }^{3}$ \\ Cássia Silva Miranda ${ }^{4}$ \\ Ivete Vieira Gomes ${ }^{5}$
}

RESUMO: O risco ocupacional biológico para os trabalhadores da saúde é um assunto muito discutido nas últimas décadas. Entretanto, os registros dos acidentes com material biológico ocorridos nas unidades de saúde não retratam a real situação. O objetivo deste estudo foi identificar o número de atendimento para acidente profissional com material biológico em um hospital de doenças infectocontagiososas e a origem dos encaminhamentos. Os dados foram obtidos por meio dos prontuários dos acidentes profissionais, no ano de 2003. Foram realizadas 5768 consultas. Destas, 621 (10,76\%) de acidente profissional, sendo 25 (4,03\%) do próprio hospital e 596 (95,97\%) de outros serviços. Verifica-se que há encaminhamento de profissionais oriundos de grandes serviços, considera-se importante estruturar os serviços de saúde para otimizar o atendimento do profissional na unidade de origem.

PALAVRAS-CHAVES: Acidentes de trabalho; Riscos ocupacionais; Notificação de acidentes de trabalho.

ABSTRACT: The occupational risk for the health's workers is a subject discussed in the last decades. However, the professional accident involving biological material's records in the health's units don't describe the real situation. The purpose of this article is to identify the number of attending of professional accident involving biological material and the source of the leading. The data were obtained by the professional accident's handbooks in 2003. The hospital had 5768 appointments. Among these, 621 (10,76\%) were about professional accident, 25 $(4,03 \%)$ of this amount came from the own hospital and $596(95,97 \%)$ from other services. The article verified that workers proceeding from big services are leaded to the hospital evaluated. It's important to structure health's services to optimize the worker's attending in the original's unit.

KEYWORDS: Occupational accidents; Occupational risk; Occupational Accidents registry.

RESUMEN: El riesgo ocupacional biológico para los trabajadores de la salud viene siendo un asunto muy discutido en las últimas décadas. Mientras tanto, los registros de accidentes con material biológico ocurridos en las unidades de salud no retratam la real situación. Los objetivos del estudio fue identificar el número de atendimientos para accidentes profesionales con material biológico en un hospital de enfermedades infectocontagiosas y el origen de los encaminamientos. Los datos de los accidentes profisionales fueron obtenidos por medio de las histórias clínicas en año 2003. Fueron realizadas 5768 consultas. De las cuales 621 (10,76\%) del propio hospital y $596(95,97 \%)$ de otros servicios. Se verifica que hay encaminamientos de profisionales oriundos de grandes servicios, se considera importante estructurar los servicios de salud para que el atendimiento del profesional sea óptimo en la unidad de origen.

PALABRAS CLAVES: Accidentes de Trabajo; Riesgos laborales; Notificación de Accidentes del Trabajo.

\footnotetext{
${ }^{1}$ Enfermeira do Serviço de Controle de Infecção Hospitalar (SCIH) do Hospital Anuar Adad (Hospital de Doenças Tropicais - HAA/HDT) e do Centro de Material e Esterilização - Hospital das Clínicas da Universidade Federal de Goiás (UFG). Especialista em Controle de Infecção

Hospitalar. Mestranda do Programa de Pós Graduação - Nível Mestrado da Faculdade de Enfermagem - UFG, liliankellylopes@hotmail.com

${ }^{2}$ Enfermeira. Doutora em Enfermagem. Professor Adjunto da Faculdade de Enfermagem - UFG. anaclara@fen.ufg.br

${ }^{3}$ Enfermeira do Centro de Material e Esterilização - HAA/HDT. sirlene.damando@ig.com.br

${ }^{4}$ Médica Infectologista do SCIH/HAA/HDT.

${ }^{5}$ Enfermeira do SCIH/HAA/HDT.
} 


\section{INTRODUÇÃO}

O risco ocupacional para os trabalhadores da saúde é um assunto muito discutido nas últimas décadas, principalmente com enfoque na soroconversão para HIV, HBV e HCV. Entretanto, os registros dos acidentes com material biológico ocorridos nas unidades de saúde não retratam a real situação (CDC, 2003).

Nos hospitais dos Estados Unidos, os Centers for Disease Control and Prevention (CDC, 2001), estimam que ocorra mais de 380.000 acidentes por ano, com material perfurocortante. Destes, $61 \%$ são causados por dispositivos com agulhas ocas. Acrescenta ainda, que outro grande número de acidentes ocorrem em instituições de saúde não hospitalares, onde o controle é praticamente impossível de se realizar. São evidentes as falhas das notificações de acidentes profissionais e as instituições assistentes (DOEBBELING et al, 2003).

No Brasil estima-se que a ocorrência de subnotificação seja ainda maior, pois existem poucas unidades estruturadas para atendimento e notificação de acidentes profissionais com material biológico e com um com programa de educação em serviço com o objetivo de sensibilizar os profissionais da saúde quanto à importância da notificação e acompanhamento dos casos e os riscos a que estão expostos após o acidente.

BINDER \& CORDEIRO (2003) realizaram um estudo sobre a subnotificação dos acidentes de trabalho no Estado de São Paulo. Os dados foram levantados por amostragem da população, realizando visitas domiciliares. Os resultados mostraram que $51,4 \%$ dos acidentes foram notificados com o preenchimento da Comunicação de Acidente de Trabalho (CAT) quando comparado com os registros obtidos no INSS, onde encontrou apenas $22,4 \%$ dos acidentes registrados. Mesmo sendo obrigatória a notificação para os funcionários que possuem vínculo empregatício regidos pela Consolidação das Leis Trabalhistas (CLT) foi detectado a subnotificação dos casos de acidentes de trabalho. Isto não difere dos acidentes profissionais com material biológico visto ter a mesma obrigatoriedade de notificação quanto ao CAT para os vínculos empregatícios privados.

Outros aspectos contribuem para a grave situação de não comunicação dos acidentes por parte dos profissionais acidentados. Dentre os vários fatores intervenientes observa-se à inexistência de serviço de atendimento ao profissional acidentado na unidade de origem, o medo de demissão nas unidades de saúde particulares, medo de saber o estado sorológico, medo da soroconversão, entre outros. Segundo Brandão (2000) apud MARZIALE et al (2004,p. ) "o acidente com material biológico pode ter repercussões psicoemocionais, levando a mudanças nas relações sociais, familiares e de trabalho", induzindo o profissional acidentado a um jogo de conflitos internos e externos, causando grande angustia e stress.
No Estado de Goiás a realidade não é diferente. Os acidentes com material biológico são notificados e acompanhados pelo Serviço de Controle de Infecção Hospitalar (SCIH) ou Serviço de Saúde e Medicina do Trabalho (SESMT). Os locais que não dispõem destes serviços encaminham seus profissionais para outros serviços que disponham do atendimento ou 0 profissional fica sem assistência.

O acidente com material biológico é considerado uma urgência médica, sendo indicado o atendimento o mais precoce possível. Os CDC (2001), GERBERDING (2003) e BRASIL (2004) recomendam que a quimioprofilaxia para acidente profissional com material biológico contaminado com sangue ou fluidos corpóreos de paciente fonte sabidamente HIV ou desconhecida seja iniciada até duas horas após o acidente, visto a sua eficácia ser melhor observada. Para que se possa seguir esta orientação o atendimento deve ocorrer o mais próximo do local de origem do acidente, sendo portanto necessário que cada unidade tenha o programa de atendimento instituído e funcionando para minimizar o tempo entre o momento do acidente e o atendimento.

A capital do Estado de Goiás dispõe de uma unidade de referência para doença infectocontagiosa, onde possui atendimento para acidente profissional com material biológico da própria unidade. Entretanto, observa-se uma alta demanda de atendimento de profissionais oriundos de outras unidades de saúde da capital e do interior do Estado, tanto da rede privada como da pública, levando a uma lacuna de tempo entre 0 momento do acidente e 0 primeiro atendimento.

Este trabalho teve por finalidade conhecer o atendimento de acidentes profissionais em unidade de doenças infectontagiosas, no ano de 2003.

\section{OBJETIVOS}

- Levantar o número de atendimento a acidente profissional, do HAA/HDT, no ano de 2003.

- Verificar o número de acidentes profissionais do HAA/HDT, no ano de 2003.

- Identificar o número de acidentes profissionais das unidades que utilizam o serviço do HAA/HDT.

\section{MÉTODO}

Estudo descritivo, retrospectivo sobre os atendimentos a trabalhares vítimas de acidentes com material biológico, realizado em uma unidade de doenças infectocontagiosas, de referência para o Estado de Goiás, localizada no município de Goiânia GO.

Os dados foram coletados realizando-se o levantamento de prontuário de todos os acidentes profissionais com material biológico atendidos no ano de 2003, após autorização prévia da instituição local e aprovação em comitê de ética em pesquisa para seres humanos, observando os aspectos ético-legais da norma vigente. 
A coleta de dados foi realizada por meio de preenchimento de uma planilha, onde foram identificado dados quanto ao número de atendimento geral de acidente profissional, o número específico de atendimento de acidentes profissional da própria unidade e de outras unidades, sendo registrado o nome das unidades de origem onde ocorreu o acidente profissional.

Os dados foram analisados e interpretados a luz das práticas norteadoras para $\mathrm{o}$ atendimento com acidente profissional com material biológico, referencial teórico acerca de condutas imediatas frente ao acidente e medidas de biossegurança preconizadas pelo CDC e Ministério da Saúde do Brasil e Secretaria Estadual da Saúde do Estado de Goiás (CDC, 2003; BRASIL, 2004; GOIÁS, 2003).

\section{RESULTADOS E DISCUSSÃO}

No ano de 2003, foram atendidas no ambulatório de urgência 5768 consultas. Destas, 621 $(10,76 \%)$ foram de acidente profissional com material biológico. Dos acidentes atendidos, $25(4,03 \%)$ foram da própria unidade. Obtivemos $596(95,97 \%)$ consultas de outras unidades, como mostra o tabela $n^{\circ} 1$.

TABELA 1 - Atendimento Geral do Ambulatório de Urgência do HAA/HDT, 2003.

\begin{tabular}{lc|c}
\hline \multirow{2}{*}{ ESPECIFICAÇÃO DO ATENDIMENTO } & \multicolumn{2}{c}{ ATENDIMENTO } \\
\cline { 2 - 3 } & $\mathrm{N}^{\circ}$ & $\%$ \\
\hline PACIENTES ATENDIDOS & 5147 & 89,24 \\
PROFISSIONAIS ATENDIDOS & 621 & 10,76 \\
ATENDIMENTO GERAL & 5768 & 100,00 \\
\hline
\end{tabular}

As diretrizes frente à Profilaxia Pós-exposição Ocupacional para HIV (PEP) normatizam que: "Quando indicada, a PEP deverá ser iniciada o mais rápido possível, idealmente, nas primeiras horas após $\circ$ acidente. Estudos em animais sugerem que a quimioprofilaxia não é eficaz, quando iniciada 24 a 48 horas após a exposição. Recomenda-se o prazo máximo, para início de PEP, seja de 72 horas após o acidente" (BRASIL, 2004, p.12 e 13).
A unidade local atende uma grande clientela de acidentes profissionais oriundas de outras unidades, como mostra o tabela $\mathrm{n}^{\circ} 2$, onde se observa 25 $(4,03 \%)$ dos atendimentos são da unidade local e 596 $(95,97 \%)$ são de outras unidades. O número sugere uma deficiência no atendimento com material biológico nas unidades de origem, o que pode significar para o profissional uma lacuna de tempo entre o momento do acidente e o primeiro atendimento.

TABELA 2 - Atendimento de Acidente Profissional no HAA/HDT, 2003.

\begin{tabular}{lc|c}
\hline \multirow{2}{*}{ ATENDIMENTO A ACIDENTE PROFISSONAL } & \multicolumn{2}{c}{ ATENDIMENTO } \\
\cline { 2 - 3 } & $\mathrm{N}^{\circ}$ & $\%$ \\
\hline Unidade Local & 25 & 4,03 \\
Outras Unidades & 596 & 95,97 \\
Total & 621 & 100,00 \\
\hline
\end{tabular}

$O$ atendimento frente a acidente profissional deve ser gratuito e imediato para 0 trabalhador acidentado, de acordo com as diretrizes do Ministério da Saúde: "Os medicamentos para quimioprofilaxia, vacina para hepatite $B$ e a imunoglobulina hiperimune para hepatite $B$ devem ser disponibilizados pelos locais de trabalho públicos ou privados... As unidades hospitalares privadas deverão ter os medicamento de PEP e vacina para hepatite $B$ adquiridos sob suas expensas" (BRASIL, 2004, p.36 e 37).
As unidades de saúde precisam implantar um Programa de Atendimento ao Profissional Acidentado, aumentando assim a efetividade e eficácia da quimioprofilaxia pós-exposiação e proporcionar maior segurança e confiança para o trabalhador.

As unidades de saúde foram categorizadas quanto ao órgão que as mantêm. Observa-se que 261 $(43,49 \%)$ dos atendimentos foram encaminhados de serviços da rede privada e $190(31,88 \%)$ da rede pública, conforme mostra o tabela $n^{\circ} 3$. 
Revista Eletrônica de Enfermagem, v. 06, n. 03, p. 324-329, 2004. Disponível em www.fen.ufg.br

TABELA 3 - Atendimento de Acidente Profissional de outras Unidades quanto ao Órgão Mantenedor, 2003

\section{ÓRGÃO MANTENEDOR}

\begin{tabular}{|c|c|c|}
\hline & \\
\hline & $\mathrm{N}^{\circ}$ & $\%$ \\
\hline Rede Privada & 261 & 43,79 \\
\hline Rede Pública & 190 & 31,88 \\
\hline Ignorado & 145 & 24,33 \\
\hline Total & 596 & 100,00 \\
\hline
\end{tabular}

TIPPLE et al (2003), desenvolveram um estudo com a finalidade de construir um Ficha de Notificação de Acidente com Material Biológico para os serviços de odontologia. Solicitaram para cada um dos 26 hospitais de grande e médio porte em Goiânia um exemplar da Ficha de Notificação de Acidente com Material Biológico utilizada na instituição. Apenas 12 unidades forneceram o exemplar. Os dados deste estudo sugerem a não existência de Programa de Atendimento de Acidente Profissional com Material Biológico na maioria destas instituições.

$\mathrm{Na}$ tabela 4 , mostra várias unidades divididas em pequeno, médio e grande porte, da cidade de Goiânia e interior. Dentre elas, algumas possuem $\mathrm{CClH}$ e $\mathrm{SClH}$ com Programa de Atendimento para Acidente Profissional com Material Biológico. Os dados não diferem dos achados de TIPPLE et al (2003), sugerindo o desconhecimento dos funcionários das unidades quanto ao fluxo de atendimento para acidente com material biológico instituído dentro das unidades ou a normatização interna das instituições é o encaminhamento de seus profissionais acidentados com material biológico para a unidade ou ainda inexiste qualquer tipo de atendimento.

O ambiente laboral deve ser seguro para a atuação de seus profissionais e a existência de um atendimento rápido frente a um acidente profissional dentro da própria unidade está incluso nas normativas para precauções padrão e Recomendações para Atendimento e Acompanhamento de Exposição Ocupacional a Material Biológico: HIV e Hepatites B e C, instituídas pelos CDC e Ministério da Saúde, proporcionando ao profissional maior segurança e confiança na instituição (CDC, 2003; BRASIL, 2004).

TABELA 4 - Atendimento de Acidente Profissional de Outras Unidades no HAA/HDT, 2003.

\begin{tabular}{lc|c}
\hline \multicolumn{1}{c}{ ESTABELECIMENTO DE SAÚDE } & \multicolumn{2}{c}{ ATENDIMENTO } \\
\cline { 2 - 3 } & $\mathbf{N}^{\circ}$ & $\%$ \\
\hline Ignorado & 145 & 24,33 \\
\hline Hospitais e Maternidades Particulares, de Grande e Médio Porte de Goiânia e Entorno & 142 & 23,83 \\
\hline Unidades de Saúde Municipais de Goiânia & 40 & 6,71 \\
\hline Unidades de Saúde Municipais do Interior & 39 & 6,54 \\
\hline Serviços Particulares Odontológicos de Goiânia & 33 & 5,54 \\
\hline Hospitais e Maternidades Públicas, de Médio e Grande Porte, de Goiânia e Entorno. & 32 & 5,37 \\
\hline Hospitais e Maternidades Particulares, de Pequeno Porte, de Goiânia e Entorno. & 32 & 5,37 \\
\hline Faculdade de Odontologia & 18 & 3,02 \\
\hline Unidades de Saúde Municipais de Aparecida de Goiânia & 14 & 2,35 \\
\hline Clínicas Particulares Psiquiátricas de Goiânia & 14 & 2,35 \\
\hline Clínicas Particulares de Goiânia & 12 & 2,01 \\
\hline Serviços de Coleta de Lixo de Goiânia e Aparecida de Goiânia & 12 & 2,01 \\
\hline Banco de Sangue & 10 & 1,68 \\
\hline Hospital e Maternidade Pública de Pequeno Porte de Goiânia e Entorno & 8 & 1,34 \\
\hline Serviços de Oftalmologia & 8 & 1,34 \\
\hline Laboratório de Análise Clínica Particular & 8 & 1,34 \\
\hline Grupo de Resgate do Corpo de Bombeiro de Goiânia & 6 & 1,00 \\
\hline Agência Prisional & 4 & 0,67 \\
\hline Serviços Particulares de Hemodiálise & 4 & 0,67 \\
\hline Serviços Públicos Odontológicos Goiânia & 3 & 0,50 \\
\hline Serviços Públicos Odontológicos de Outros Municípios & 3 & 0,50 \\
\hline Faculdade de Terapia Ocupacional & 2 & 0,34 \\
\hline Clínica Psiquiátrica Pública & 2 & 0,34 \\
\hline Laboratório de Análise Clínica Público & 2 & 0,34 \\
\hline Farmácia de Silvânia & 1 & 0,17 \\
\hline
\end{tabular}


Revista Eletrônica de Enfermagem, v. 06, n. 03, p. 324-329, 2004. Disponível em www.fen.ufg.br

\begin{tabular}{lc|c}
\hline Estabelecimento de Recilclagem de Lixo & 1 & 0,17 \\
\hline Faculdade de Biomedicina & 1 & 0,17 \\
\hline Total & 596 & 100,00 \\
\hline
\end{tabular}

Outro aspecto observado foi à deficiência dos registros dos acidentes atendidos na unidade local, pois $145(24,33 \%)$ dos acidentes não foram identificados quanto à unidade de origem, visto não constarem relatos nem no prontuário e nem na Ficha de Atendimento a Acidente Profissional com Material Biológico.

No panorama geral dos atendimentos realizados no ano de 2003 observa-se o atendimento de um profissional encaminhado de uma farmácia, um profissional encaminhado de uma em empresa que faz reciclagem do lixo, doze atendimentos encaminhados da empresa que coleta o lixo comum e 57 atendimentos oriundos de serviços odontológicos públicos e privados. Apresenta-se de forma otimista um crescente despertar de outras categorias profissionais para os riscos a que estão expostos durante as execuções de suas funções. Entretanto, um programa de educação em biossegurança em nível Estadual é de extrema importância para atingir o objetivo de sensibilizar os profissionais expostos a este tipo de risco, quanto aos cuidados primordiais para o controle da soroconversão para HIV e Hepatites B e C.

Faz-se necessário educar a população em geral quanto aos cuidados necessários para o descarte de materiais perfurocortantes, pois ocorre a utilização de materiais biológicos pela população, visto existir pessoas insulinodependentes, usuários de drogas e outros tipos de necessidades presentes em ambientes não hospitalares, que geram resíduos infectantes de risco para os coletores de resíduos. GARCIA \& ZANETTI-RAMOS (2004), fazem considerações quanto a natureza do lixo domiciliar, levando a ter uma nova política de segregação para os mesmos. $O$ acondicionamento adequado é de suma importância para evitar a exposição dos profissionais que realizam a coleta dos resíduos.

Com novas abordagens para a Segregação e Acondicionamento dos Resíduos de Serviços de Saúde e da comunidade, tratado pela ANVISA na RDC $n^{\circ} 33$ (BRASIL, 2003) a reciclagem dos resíduos está sendo enfocada como medida de diminuir os resíduos gerados pelas instituições e a população em geral. Detectamos um profissional acidentado que realiza coleta e recicla resíduos. Este indivíduo encontra-se expostos a risco de acidentes quando as unidades geradoras dos resíduos não possuem uma política de Gerenciamento de Resíduos eficaz.

\section{CONCLUSÃO}

A unidade de Doenças Infectocontagiosas atendeu, no ano de 2003, uma quantidade significativa de acidentes com material biológico na unidade de emergência, sugerindo uma confiança dos profissionais no atendimento oferecido pela instituição.

O número atendimentos encaminhados de outras unidades foi maioria absoluta, sugerindo a inexistência, ineficiência e ineficácia dos Programas de Atendimento de Acidente Profissional com Material Biológico.

Novas categorias profissionais foram identificadas em exposição a risco biológico, procurando atendimento pós-exposição com material biológico. Existe uma preocupação e sensibilização de outras categorias de serviços de saúde extra-hospitalar, na política de risco biológico.

Existe uma deficiência nos registros dos acidentes atendidos na unidade de Doenças Infectocontagiosa sugerindo a necessidade de treinamento e supervisão dos profissionais que fazem o atendimento na unidade quanto à importância do registro dos acidentes.

Conclui-se que existe uma deficiência no atendimento de Acidente Profissional com material Biológico no Estado de Goiás, que necessita de investimentos políticos, financeiros e compromisso profissional, para que haja a resolução ou minimização das dificuldades levantadas.

Os órgãos normatizadores e fiscalizadores necessitam investir na existência de Programas de Atendimento a Acidente com material biológico dentro das Unidades de Saúde, para minimizar e controlar o risco de soroconversão para HIV e Hepatites B e C. A deficiência no atendimento e acompanhamento dos acidentes profissionais com material biológico impossibilita o conhecimento da situação real de soroconversão ou eficácia do Programa de Atendimento atual, bem como gera a insegurança dos profissionais frente a uma exposição ocupacional.

As unidades que possuem Comissão de Controle de Infecção Hospitalar e Serviço de Controle de Infecção Hospitalar e Programa de Atendimento a Acidente com Material biológico necessitam atualizar os profissionais saúde (médico, enfermeiros, odontólogos, auxiliares de enfermagem e etc) quanto às rotinas de atendimento pós-acidente com material biológico e quanto à importância da notificação e acompanhamento do profissional, não deixando de enfocar todas as medidas de precauções padrão.

As unidades que possuem Comissão de Controle de Infecção Hospitalar e Serviço de Controle de Infecção Hospitalar e não possuem um Programa de Atendimento a Acidente com Material biológico necessitam instituí-lo com urgência dentro da instituição para diminuir o tempo entre o acidente e o atendimento, minimizando o estresse presente na situação de risco vivenciada pelo profissional acidentado.

Uma avaliação criteriosa deve ser feita quanto à implantação de uma unidade de referência para atendimento a acidente profissional com material biológico na cidade de Goiânia, constituída de uma equipe multiprofissional para assistir os casos de profissionais que não atuam dentro de unidade de saúde, realizando busca ativa dos casos faltosos às 
consultas de retorno, possibilitando assim, o acompanhando dos acidentados até a alta por cura ou soroconversão.

As unidades de Cidades do interior do Estado de Goiás necessitam implantar um Programa de Atendimento a Acidente Profissional com Material Biológico para agilizar 0 atendimento dos seus profissionais.

Oferecendo segurança e eficácia.

O Sistema Único de Saúde encontra-se dentro do processo de municipalização, dando gestão plena para unidades que atendem os critérios necessários. Dentro do Programa verbas são destinadas para a área da saúde, incluindo a saúde do trabalhador. Portanto, os gestores municipais e estaduais necessitam incluírem em seus planejamentos estratégicos o Programa de Atendimento ao Profissional e destinar verbas para a sua implantação e implementação nos estabelecimentos de saúde existentes em seus municípios.

\section{REFERÊNCIAS BIBLIOGRÁFICAS}

BINDER, M. C. P. CORDEIRO, R. Sub-registro de Acidentes do Trabalho em localidade do Estado de São Paulo, 1997. Rev. Saúde Pública. v. 37 n. 4, p. 409-416, 2003.

BRASIL, Ministério da Saúde. Plano de Gerenciamento dos Resíduos dos Serviços de Saúde Resolução n 33/2003. Brasília, DF, 2003.

BRASIL, Ministério da Saúde. Recomendações para Atendimento e Acompanhamento de Exposição Ocupacional a Material Biológico: HIV e Hepatites B e C. Brasília, DF, $2004.25 \mathrm{p}$. http://www.aids.gov.br/final/biblioteca/manual exposica o/manual acidentes.doc. Acesso em 27 de junho de 2004.

CDC - Centers for Disease Control and Prevention. Guideline for the Management of Occupational Exposure to HBV, HCV, and HIV and Recommendations for postexposure prophylaxis. MMWR. v. 50, $\mathrm{n}^{\circ}$ RR11, p.1-42, 2001. http://www.cdc.gov/mmwr/preview/mmwhtml/rr5011a1. htm. Acesso em 15 de junho de 2003.

$\overline{\mathrm{CDC}}$ - Centers for Disease Control and Prevention. Tramission of Hepatitis $B$ and $C$ Viruses in Outpatient Settings, New York, Oklahoma, and Nebraska, 20002002. MMWR. v. 52, $\mathrm{n}^{\circ}$ 38, p. 901-906, 2003. http://www.cdc.gov./mmwr/preview/mm. Acesso em 21 de maio de 2004.

GARCIA, L. P., ZANETTI-RAMOS, B. G. Gerenciamento dos Resíduos de Serviços de Saúde: uma questão de Biossegurança. Cad. Saúde Pública, v. $20, n^{\circ} 3$, p. $744-752,2004$

GOIÁS. Secretaria de Estado de Saúde. Programa de Prevenção ao Acidente Profissional com Material Biológico. Goiânia, 2003.

DOEBBELING, B. N., VAUGHN, T.E., MCCOY, K.D., BEEKMANN, S.E., WOOLSON, R. F., FERGUSON, K. J., TORNER, J. C. Percutaneous Injury, Blood Exposure, and Adherence to Standard Precautions: Are Hosptial-Based Health Care providers Still at Risk? CID, v.37, p. 1006-1013, 2003.

GERBERDING, J. L., Occupational Exposure to HIV in Health Care Settings. N. Engl. J. Med. v. 348, $n^{\circ}$ 9, p. 826-833, 2003. Disponível: http://www.nejm.org by Boaventura B. de Queiroz MD. Capturado em 24 de Junho de 2003.

MARZIALE, M.aria Helena Palucci, NISHIMURA, Karina Yukari Namioka, FERREIRA, Mônica Miguel. Riscos de Contaminação Ocasionados por Acidentes de Trabalho com Material Perfurocortante Entre Trabalhadores de Enfermagem. Revista Latino-am Enfermagem, São Paulo, v. 12, no 1, p. 36-42, 2004. http://www.scielo.br/pdf/rlae/v12n1/v12n1a06.pdf. Capturado em 27/06/2004.

TIPPLE, A. F. V., PAIVA, E. M. M., et al. Ficha de Notificação de Acidentes com Material Biológico Aplicada à Odontologia. Robrac, v.12, n. 33, p. 45-50, 2003.

Texto recebido em 30/08/2004.

Publicação aprovada em 10/12/2004 